\title{
DETERMINAÇÃO DO CAMPO DE VELOCIDADE EM UMA SEÇÃO RISER PELA TÉCNICA DE VELOCIMETRIA POR IMAGEM DE PARTÍCULA
}

\author{
A. R. FERNANDO ${ }^{1}$, G. J. CASTILHO ${ }^{1}$ \\ ${ }^{1}$ Universidade Estadual de Campinas, Faculdade de Engenharia Química \\ E-mail para contato: Andre.rogeriofernando@gmail.com
}

\begin{abstract}
RESUMO - Leitos fluidizados circulantes (LFC) são amplamente utilizados na indústria de processos, principalmente na indústria de refino de petróleo para se realizar o processo de craqueamento catalítico fluido. Dentre as partes que constituem uma unidade LFC destaca-se o Riser, parte onde ocorrem de fato as reações de craqueamento catalítico. Realizar a análise do comportamento fluidodinâmico no Riser é de extrema importância para o entendimento e a otimização do processo. Dentre as técnicas de análise utilizadas para se medir grandezas neste tipo de sistema particulado destaca-se a PIV (velocimetria por imagem de partícula), uma técnica robusta e não intrusiva que pode determinar campos de velocidade e estimar a concentração de um fluido em escoamento com grande precisão. Neste trabalho foi utilizada a técnica Tomographic PIV (ou tomoPIV), uma variação do PIV que permite a obtenção dos campos de velocidade em três dimensões para se analisar um escoamento em uma seção Riser de um LFC montado no laboratório de processos de meio porosos da Faculdade de Engenharia Química da Universidade Estadual de Campinas.
\end{abstract}

\section{INTRODUÇÃO}

Leitos Fluidizados circulantes (LFCs) são equipamentos amplamente utilizados em processos de mistura, secagem e reações catalíticas. Sua larga utilização se deve ao fato de poderem oferecer altas taxas de troca de calor e massa, alta agitação das partículas sólidas, e a possibilidade de utiliza-los em processos contínuos e automatizados. Uma de suas aplicações mais importantes está na indústria de petróleo, onde os LFCs são utilizados no processo de craqueamento catalítico fluido (Castilho, 2011). Devido a sua importância, os LFCs são objetos de intensa investigação, e dentre as diversas técnicas de análise usadas para investigar as características de escoamentos nos LFCs, destaca-se a PIV.

Segundo Adrian (1991) a técnica PIV é considerada uma técnica de análise robusta e não intrusiva que tem como objetivo obter campos de velocidade instantâneos de um fluido em movimento. A técnica utiliza partículas traçadoras misturadas ao fluido analisado, estas partículas são iluminadas por um laser pulsante, espalhando a luz em uma lente fotográfica CCD. As imagens obtidas são então enviadas para um computador, onde são analisadas.

Existem diversas variações da técnica PIV, as principais são o PIV simples, o stereoPIV e o tomo-PIV. Dentre estas três, o tomo-PIV se destaca como a mais completa. Como definiu Elsinga (2006), o tomo-PIV faz uso de visualizações simultâneas, com perspectivas 


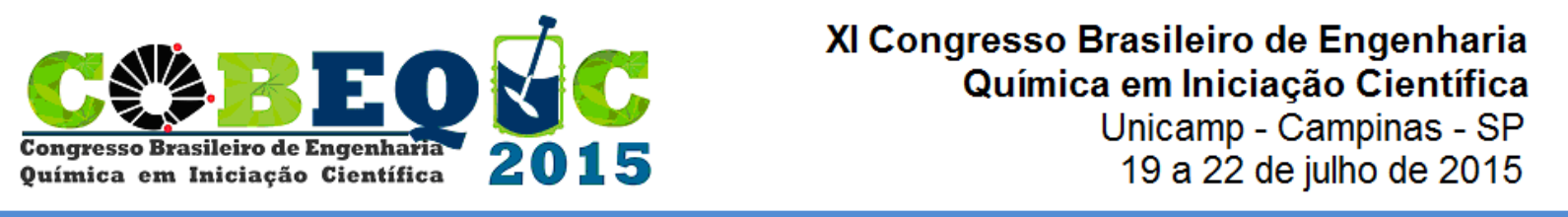

diferentes das partículas iluminadas pelo laser, e realiza uma reconstrução e tomográfica para se obter uma distribuição de luz em 3D associada a distribuição das partículas traçadoras no volume investigado. O deslocamento das partículas é obtido através da aplicação de uma relação cruzada 3D da distribuição das partículas reconstruídas. Este método é exemplificado na Figura 1. O tomo-PIV é considerada uma técnica complexa, mas se aplicada corretamente permite a obtenção de resultados altamente precisos.

Figura 1 - Princípio de funcionamento do tomo-PIV (Elsinga, 2006).

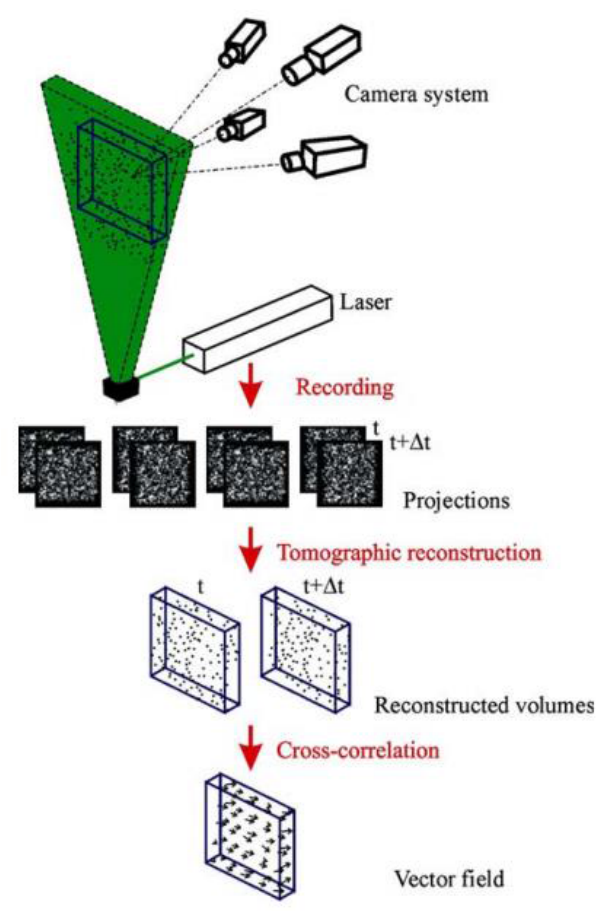

\section{MATERIAIS E METODOS}

Neste trabalho realizou-se a analise do campo de velocidade da seção riser utilizandose a técnica tomo-PIV, com 4 câmeras CCD e um sistema de laser Nd:YAG. Esta análise pode ser dividida em diversos passos, como ilustrado na Figura 2. O software empregado para realizar a análise foi o DAVIS 8.0.

O experimento foi realizado utilizando como fase fluida o ar e como fase particulada catalisadores de FCC. O escoamento gás-sólido foi avaliado na seção riser da unidade de leito fluidizado circulante (Figura 3). O diâmetro do tubo é de $8,2 \mathrm{~cm}$, a vazão de ar utilizada foi de $82 \mathrm{Nm}^{3} / \mathrm{h}$, o que resulta em uma velocidade média de escoamento de aproximadamente $4,5 \mathrm{~m} / \mathrm{s}$, a vazão de sólidos foi de $1,8 \mathrm{~g} / \mathrm{s}$.

Antes da gravação das imagens foi realizado um processo de calibração, uma etapa muito importante, pois define a qualidade final da análise. A calibração foi realizada utilizando-se uma placa de calibração composta de dois planos paralelos com pontos de marcação equidistantes distribuídos pela sua superfície. Utilizando imagens obtidas pelas 4 câmeras é possível definir a posição de cada câmera em relação ao alvo e assim realizar a calibração (LaVision, 2010 a). 


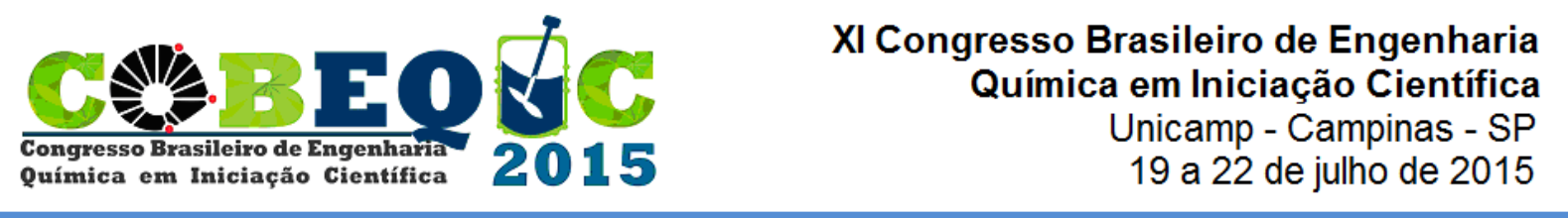

Figura 2 - Etapas para a obtenção do campo de velocidade (Adaptado de Amaral, 2012).

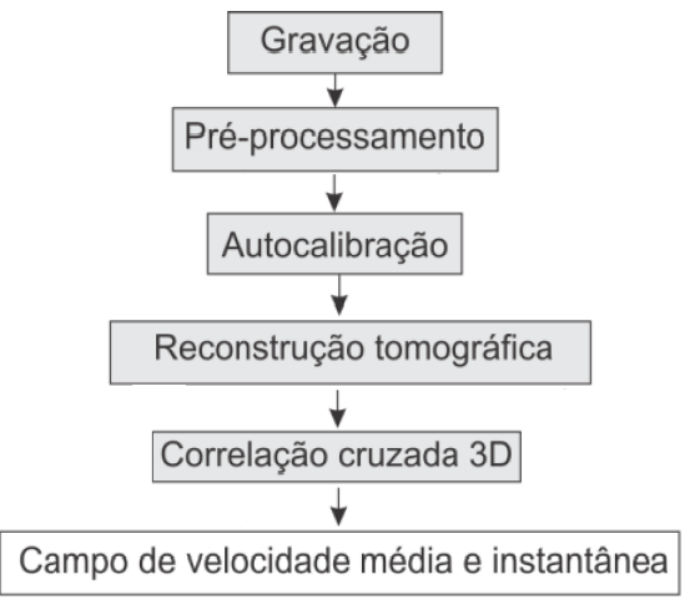

Figura 3 - Esquema da unidade LFC do LPMP/FEQ/Unicamp (Amaral 2012)

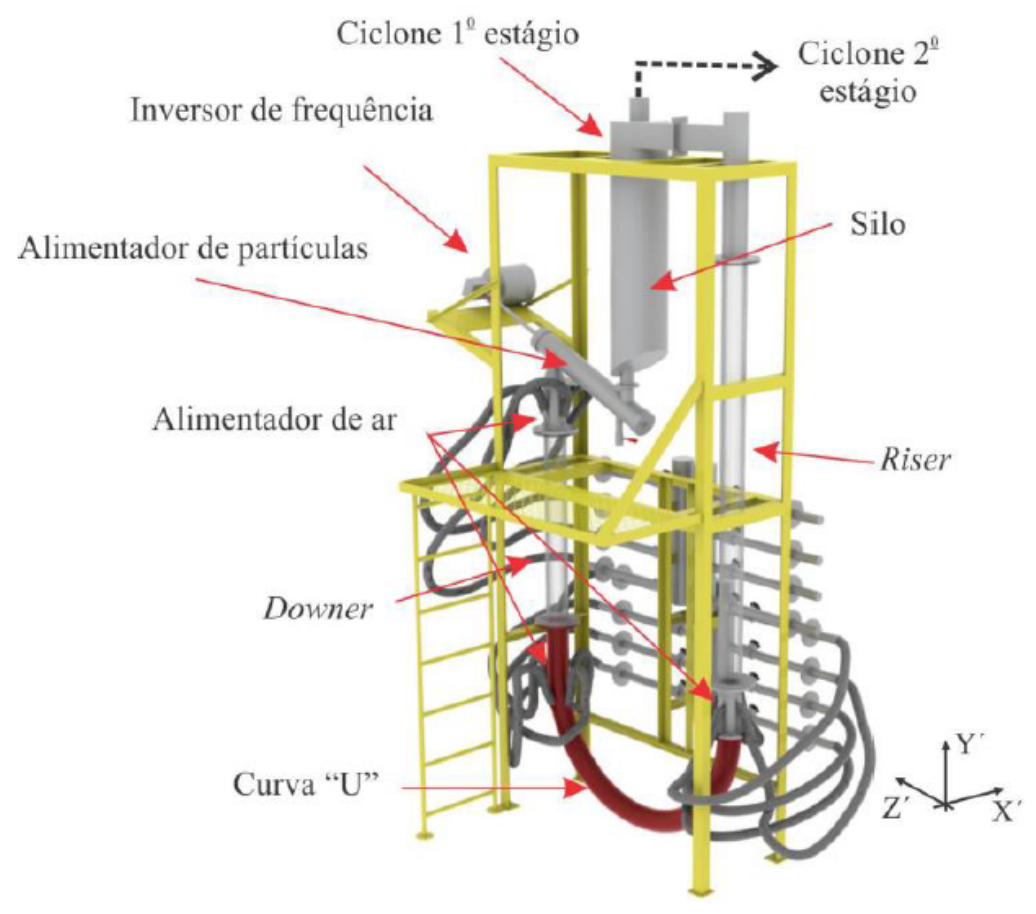

As imagens foram captadas pelas câmeras no modo de exposição dupla, ou seja, cada câmera capta duas imagens separadas por um intervalo de tempo dt, ajustado em $100 \mu \mathrm{s}$. Como análise necessita de um número grande de imagens, foram captadas 150 imagens, resultando em um tempo de gravação de aproximadamente 6 segundos. Em seguida estas imagens passaram por um pré-processamento com o objetivo de isolar o volume a ser analisado e eliminar objetos indesejáveis, como o fundo da imagem, deixando apenas as partículas iluminadas pelo laser. 
Antes de prosseguir com a analise foi realizado um procedimento opcional chamado de autocalibração, como descrito por Amaral (2013), este procedimento emprega as imagens pré-processadas para reduzir erros da reconstrução, resultantes do desalinhamento das câmeras que podem ocorrer devido a uma calibração imprecisa ou pequenos movimentos das câmeras. Antes de realizar a autocalibração os erros eram da ordem de 0,9 pixels, um erro relativamente alto. Uma calibração ideal apresenta erros menores do que 0,1 pixel (LaVision, 2010 b), no entanto deve-se notar que o volume analisado é cilíndrico, logo existe uma distorção maior da imagem resultando naturalmente em erros maiores. Após o processo de autocalibração foi possível reduzir os erros médios para aproximadamente 0,05 pixel.

Depois da autocalibração, realizou-se a reconstrução tomográfica, esta etapa utiliza a técnica MART (técnica de reconstrução algébrica multiplicativa), um método iterativo que através da resolução de equações lineares permitem a reconstrução de um volume utilizando as imagens captadas pelas câmeras (LaVision, 2010 b). O volume reconstruído na análise realizada tem as seguintes dimensões: $60 \times 30$ x $40 \mathrm{~mm}$, este volume é intencionalmente maior do que o volume iluminado pelo laser.

Com o volume reconstruído, foi aplicada a correlação cruzada para calcular os vetores, este método envolve determinar o deslocamento de pequenos grupos de vetores contidos em regiões chamadas de janelas de interrogação, gerando assim um vetor deslocamento para cada janela. Em cada janela é definido um pico de iluminação do laser, para intervalos de tempo pequenos, dois frames seguidos apresentam distribuições de luz parecidas, mas com picos levemente deslocados, assim é possível computar um vetor deslocamento para cada janela de inspeção (Amaral, 2013). Este processo está exemplificado na Figura 4.

Figura 4 - Esquema do procedimento de correlação cruzada (Amaral, 2013).

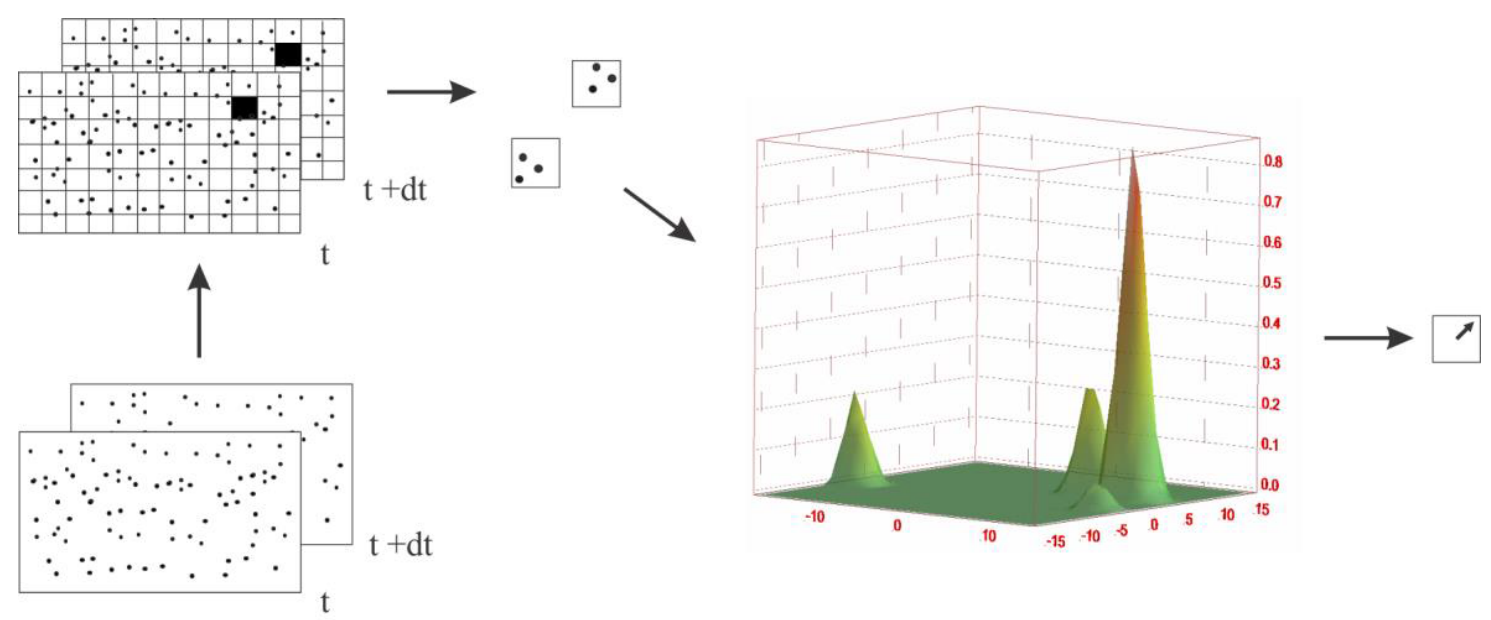

Este processo é realizado de modo interativo, diminuindo aos poucos o tamanho das janelas de interrogação para permitir maior precisão no cálculo dos vetores. O último passo da técnica foi a aplicação de filtros de pós-processamento, estes tem o objetivo de eliminar irregularidade no campo vetorial resultante, no caso analisado não houve mudanças significativas, pois o campo vetorial obtido era bastante regular. O resultado final obtido é um conjunto de imagens que mostram os campos de velocidade em 3D.

Após calcular o campo de velocidade, também é possível obter outras informações sobre o escoamento, como perfis de escoamento, velocidades por componentes $\left(\mathrm{v}_{\mathrm{x}}, \mathrm{v}_{\mathrm{y}} \mathrm{e} \mathrm{v}_{\mathrm{z}}\right)$, e 
com um processamento especifico também é possível obter um mapa da distribuição das partículas no volume analisado que pode ser usado para estimar a concentração de partículas.

\section{RESULTADOS}

A partir das imagens capturadas pelas câmeras foi realizado o pré-processamento, o resultado pode ser observado na Figura 5, observa-se que a imagem à direita já não apresenta o fundo e nem os reflexos do tubo, que podem ser observados na imagem capturada pela câmera, estes elementos provavelmente causariam problemas nas outras etapas caso não fossem eliminados das imagens.

Figura 5 - Comparação entre imagem obtida pela câmera e após o pré-processamento.

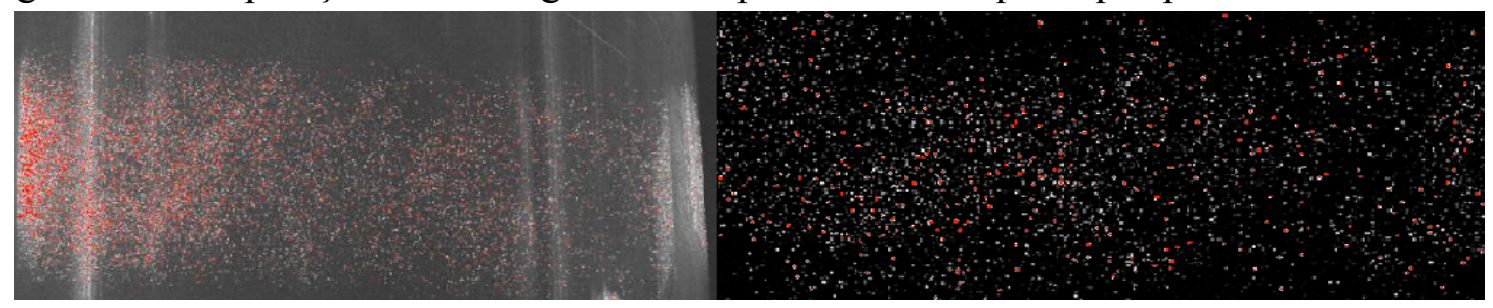

As imagens obtidas após a reconstrução e a aplicação da correlação cruzada estão representadas a seguir, a Figura 6 mostra o campo de velocidade média referente a todo o tempo de gravação, ou seja, 6 segundos, projetado em um plano $\mathrm{Z}$ específico, enquanto a Figura 7 mostra o campo de velocidade completo em 3D.

$\mathrm{O}$ plano $\mathrm{Z}$ da Figura 6 representa a região do centro do tubo da unidade riser, é possível perceber que o campo resultante é homogêneo, existem pequenas variações na direção do movimento. Ao observar a Figura 7, notam-se variações de velocidade na direção $\mathrm{z}$, mas na média as partículas se movimentam em uma trajetória quase reta na direção $\mathrm{Y}$. As cores no fundo da Figura 6 representam a velocidade média, nota-se que a velocidade na região do lado esquerdo e no centro é um pouco superior, em torno de $5,2 \mathrm{~m} / \mathrm{s}$ enquanto do lado direito é um pouco menor, próxima a $4,6 \mathrm{~m} / \mathrm{s}$, estes resultados são próximos ao esperado, já que a velocidade estimada através das medidas do rotâmetro foi de 4,5 m/s.

Figura 6 - Resultado final da análise por tomo-PIV.

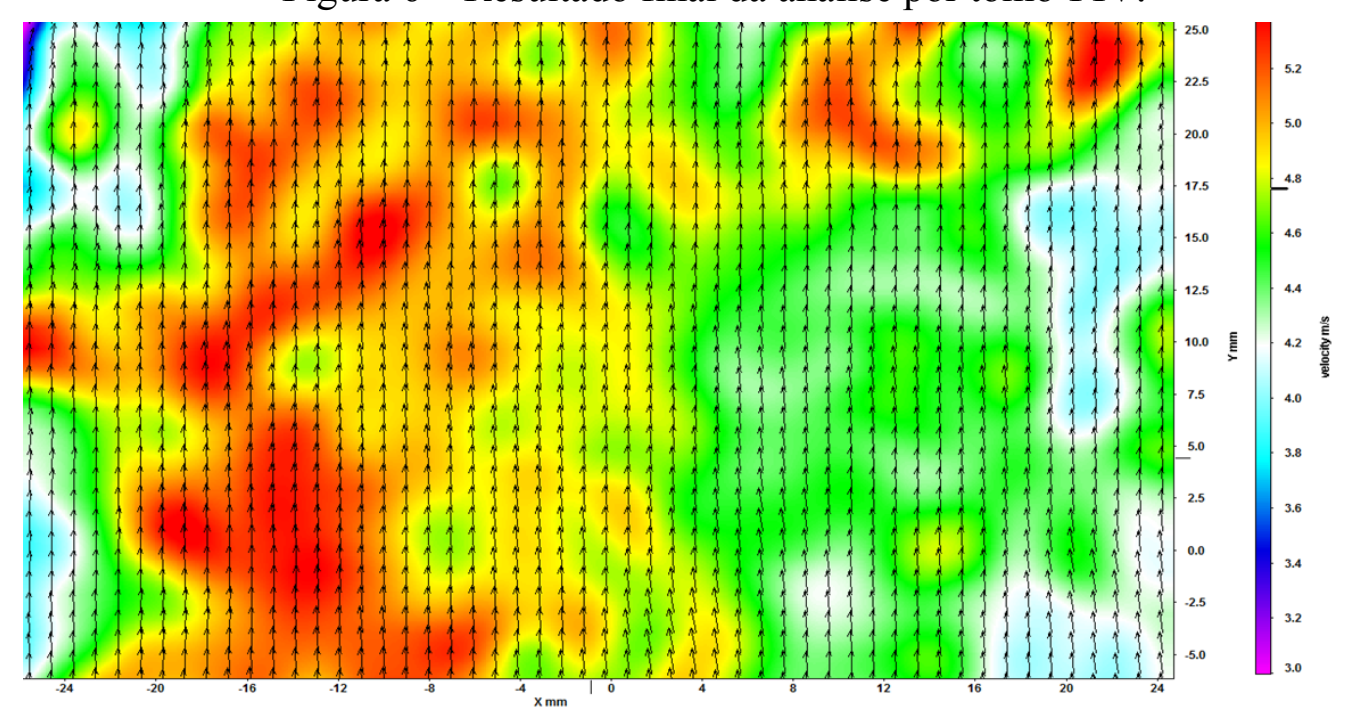


Figura 7 - Campo de velocidade em 3D.

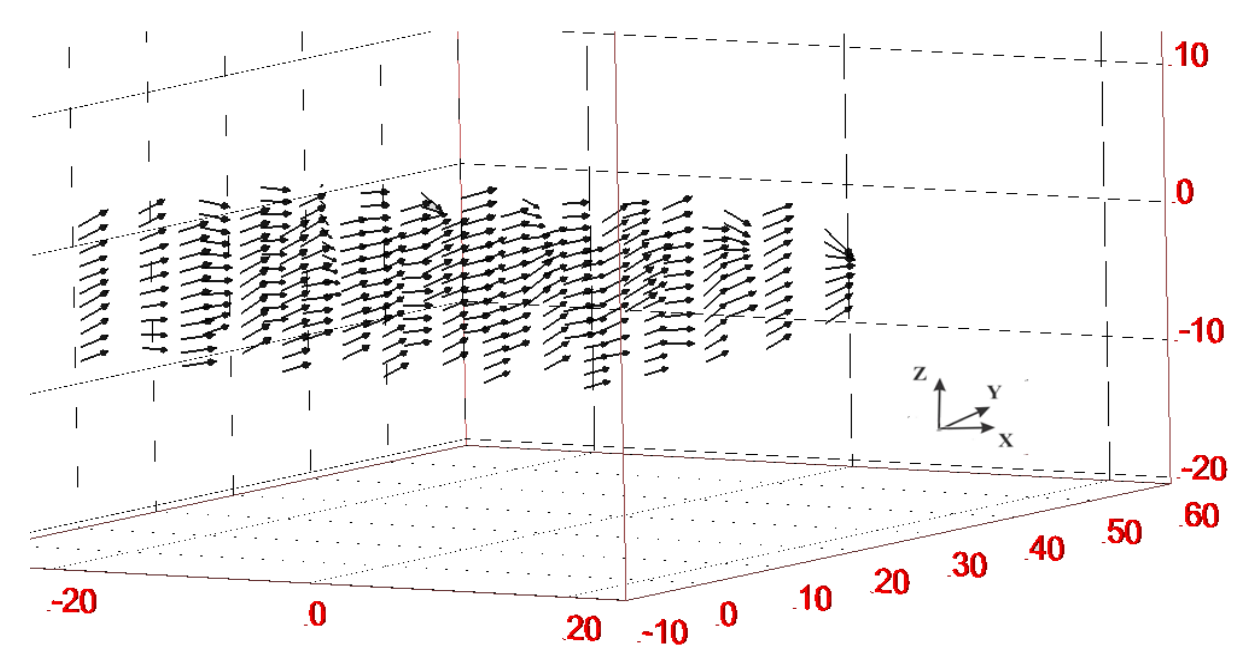

\section{CONCLUSÃO}

A aplicação da técnica tomo-PIV é um processo trabalhoso e necessita muito cuidado e um pouco de prática, pois é necessário ajustar os parâmetros de cada passo do processamento das imagens até se obter um resultado satisfatório. Apesar da complexidade da técnica a análise da seção riser foi realizada com sucesso e obteve-se um campo de velocidade homogêneo com velocidades próximas a $5 \mathrm{~m} / \mathrm{s}$, velocidade um pouco superior a velocidade calculada a partir da vazão medida no rotâmetro $(4,5 \mathrm{~m} / \mathrm{s})$, pequenas imprecisões podem ser explicadas por erros na calibração inicial e parâmetros não ideais escolhidos durante as diversas etapas do processamento, que podem implicar em imprecisões no cálculo dos vetores.

\section{REFERÊNCIAS}

ADRIAN, R. J. (1991), Particle-imaging techniques for experimental fluid mechanics, Annu. Rev. Fluid Mech., Vol. 23, 261-304.

AMARAL, R. L, Efeito da autocalibração volumétrica para PIV tomográfico no campo de velocidade em uma seção de um riser de um leito fluidizado circulante. Tese de Mestrado. Universidade Estadual de Campinas, 2013.

CASTILHO, G. J. C. Análise de Caos em Leito Fluidizado Circulante. Campinas: FEQ/UNICAMP. Tese (Doutorado),2011

ELSINGA, G. E.; SCARANO, F.;WIENEKE, B.;VAN OUDHEUSDEN, B. W. Tomographic particle image velocimetry, Exp. Fluids, Vol. 41,p. 933-947, Springer-Verlag. 2006

LAVISION. Product Manual: FlowMaster. Göttingen. 2010.

LAVISION. Product Manual: Tomographic-PIV. Göttingen. 2010. 